\title{
Entretien avec Marcel Czermak
}

\section{(2) OpenEdition \\ 1 Journals}

Édition électronique

URL : http://journals.openedition.org/conflits/467

DOI : $10.4000 /$ conflits.467

ISSN : $1777-5345$

Éditeur :

CCLS - Centre d'études sur les conflits lilberté et sécurité, L'Harmattan

Édition imprimée

Date de publication : 15 juillet 1995

ISSN : 1157-996X

Référence électronique

«Entretien avec Marcel Czermak », Cultures \& Conflits [En ligne], 18 | été 1995, mis en ligne le 15 mars 2006, consulté le 30 mars 2021. URL : http://journals.openedition.org/conflits/467 ; DOI : https:// doi.org/10.4000/conflits.467

Ce document a été généré automatiquement le 30 mars 2021.

Creative Commons License 


\section{Entretien avec Marcel Czermak}

C\&C: A plusieurs reprises les auteurs de ce numéro s'interrogent sur la validité scientifique du concept d'enfance et, notamment sur la pertinence qu'il revêt en sciences sociales. Quelle réponse peut proposer le chercheur et le clinicien que vous êtes?

Marcel Czermak<: L'homme a ce privilège parmi tous les autres animaux d'être un être prématuré. Son achèvement neurologique et biologique est en effet tardif et ses débuts sont caractérisés par une dépendance radicale de qui veille sur lui. Donc très précocement, ce rapport avec ce que l'autre lui demande ou attend de lui sera la marque définitive de sa prématurité et de sa dépendance. En outre l'enfant présente la particularité, contrairement à tous les autres animaux, d'être un être parlant ou futur parlant. Ce qui caractérise l'enfance n'est donc pas seulement un rapport de stricte dépendance biologique à son environnement. Parler d'enfance en tant qu'adulte est difficile car nous ne voyons pas ce qui s'y passe. La socialisation de l'être humain est un processus de refoulement au cours duquel on apprend à se taire sur le plan sexuel, social, etc., de manière à maintenir un rapport tempéré avec les autres. Il s'opère une sorte de transformation où les questions primitives, primordiales, tombent dans les dessous et empruntent des mécanismes divers qui sont d'ailleurs cliniques et par lesquels le sujet essaie de concilier la maintenance d'un lien social et les questions primordiales qui se sont posées à lui et qu'il a simultanément oubliées. Dès qu'un enfant naît au monde, il est immédiatement pris dans la socialisation. Avant même sa naissance tout un discours l'antécède et cadre sa place sans qu'il y puisse strictement rien. Comme adultes nous fantasmons sur ce que nous voulons de nos descendants sans avoir la moindre idée de l'incidence de ce fantasme sur l'enfant et ça c'est un problème qui échappe à proprement parler à la science. $</ p>$

C\&C : Pourtant l'enfant existe dans la cité. Comment le sociologue et le politologue pourraient-ils traiter cet « objet d'étude »?

MC. : On touche ici à la définition de l'objet d'études en sciences. Dans le cas de l'enfant, on peut au moins considérer que c'est un objet à multiples faces. En tant qu'objet du désir des siens, il est un objet qui ne se définit, après coup, qu'en fonction des valeurs qu'on place en lui. Objets d'aucun désir les enfants deviendraient fous, trop dociles ou indociles aux attentes placées en eux. Ils deviendraient psychotiques. Tels sont les pôles 
du passage de l'enfance à l'âge adulte : ordinairement, à un pôle nous avons la névrose infantile; à l'autre la névrose plus ou moins socialisée de l'adulte, séparées par une latence active qui est le processus même du passage de l'enfant à l'adulte.

C\&C. : Ne pourrait-on pas dire, en simplifiant, qu'un enfant devient adulte à partir du moment où il se tait sur un certain nombre de ses désirs?

M.C. : Certainement. Mais je voudrais évoquer à cet égard le texte freudien relatif à ce qu'on appelle après lui la période de latence. C'est l'âge où les enfants nous fichent la paix, où ils ne s'intéressent pas à ce qui se passe dans le lit des parents, où ils apprennent à être polis, à ne pas cracher à la figure des gens, à être "comme il faut ", quoi. C'est un pur effet de socialisation psychologique dans lequel nous affirmons, avec d'autres, qu'il n'y a rien de biologique. Puis vient le temps de l'adolescence, avec ses poussées pulsionnelles, biologiques cette fois, ses obligations, ses responsabilités, et tout craque. Certains enfants font l'économie de cette période de latence. Ce sont des acteurs sociaux précoces dont la socialisation primitive au titre de la névrose infantile est immédiatement opérationnelle. Je suis enclin à penser que dès qu'un gosse a dans le langage les éléments de la manoeuvre sociale, soit entre quatre et six ans, il dispose de l'essentiel pour se débrouiller dans la vie. Le plus souvent cependant l'hominisation du parlant passe par une phase de refoulement, au cours de laquelle il ne met pas en oeuvre ce qu'il est capable de mobiliser. A l'inverse, si la conjoncture s'y prête, il est capable d'une attitude, stratégiquement organisée et finalisée.

C\&C : Leur violence peut alors prendre un tour politique, mais peut-on dire par exemple qu'en raison d'une sexualité défaillante, cette violence juvénile est différente de celle des adultes comme le disent certains psychologues?

M.C. : Toute sexualité est plus ou moins « défaillante», comme vous dites, mais nous savons bien d'autre part qu'elle est un élément essentiel de la socialisation. Pour autant que l'enfant est objet de désir, toute sa sexualité se trouve impliquée. Elle génère en particulier de manière extraordinairement précoce un type de relation qui passe par la reconnaissance de soi par l'autre et dans laquelle le sujet ne se reconnaît qu'en tant que l'autre le reconnaît et lui renvoie sa propre image. Or ce rapport du petit enfant à son semblable, à l'alter ego, à son image, est d'une violence extrême. La seule chose susceptible de le pacifier est la mise en route d'une instance tierce qui va dire : «Pour que moi je vous reconnaisse il faut que vous m'obéissiez». Cette instance tierce, porteur de la loi, des symboles, de la culture, fonctionne à l'interdiction et, grâce à la parole, fait qu'on ne doit pas détruire nous-mêmes notre propre image dans le prochain. Je suis donc convaincu que la violence des enfants, politique ou non, n'est pas fondamentalement différente de celle des adultes sauf dans la conjoncture infantile primordiale où le rapport au semblable n'est réfuté par aucune instance tierce. Métaphoriquement c'est ce que révèlent certains conflits comme en ex-Yougoslavie où l'image de l'autre apparaît immédiatement mortifère. Là, en guise d'instance tierce on envoie l'ONU. Mais l'ONU n'a pas compris que dans une telle conjoncture le régime de la loi parlée ne peut être appliquée que par la force.

C\&C : La violence politique des enfants et celle des adultes s'exercent-elles selon des modalités différentes? Et si oui, cela ne tient-il pas à une différence de structure, disons, psychologique entre les uns et les autres?

M.C. : Sans aucun doute. Tous nos modèles comportementaux d'adultes sont élaborés à partir de modèles pulsionnels fondamentaux selon des modalités qui, dans la socialisation ultérieure, font la culture. Cependant dans les conflits politiques 
extrêmement aigus où les règles ne valent plus on voit réapparaitre cette espèce de sauvagerie initiale qui est celle de chacun d'entre nous. Qu'il y ait des cultures ou des civilisations qui se prêtent plus que d'autres à la brutale résurgence des mécanismes de cette primordialité humaine, c'est un tout autre problème, que je ne vois pas trop, personnellement, comment aborder.

C\&C. : N'est-ce pas dans ce cas renouer avec la théorie du «conflit primordialiste » ?. Pourriez vous dire que la méchanceté de l'adulte est un retour à l'archaïsme de l'enfant avec de la technique et éventuellement de la stratégie en plus?

M.C. : Oui, mais l'enfant aussi est tout à fait capable de jouer en bordure à la limite des transgressions. Par contre il n'est pas, d'une manière générale, susceptible, d'une attitude stratégiquement finalisée et mobilisatrice. Mais il y a des exceptions qui nous étonnent, car on répugne à reconnaître notre propre violence dans celle des gosses que nous fûmes et dont on ne sait trop si l'encadrement relève du droit ou de la thérapeutique. La violence politique juvénile signe en tout cas l'échec de l'instance tierce, de la loi impuissante à civiliser le comportement du sujet et, notamment, de border son rapport au prochain. Faute d'être encadré par des signifiants symboliques il a vocation à l'être par les murs de la prison ou de l'hôpital.

C\&C. : Quelles conclusions pourrait-on en tirer plus spécifiquement pour nos sociétés ?

M.C. : La violence politique et la délinquance de nos jeunes, notamment en banlieue, est un des problèmes majeurs de nos sociétés. Nous avons donc à réfléchir sur la manière de réintégrer dans la vie des enfants-citoyens ce qui, avec la socialisation, est une restriction de liberté, exclusive des manifestations de la violence primitive primordiale élémentaire. Avec la mise hors champ des facteurs de socialisation intra ou extrafamiliale, on a supprimé le symbolique, garant de la parole et avec les modalités du symbole c'est l'intégration qui vole en éclats. Il est donc urgent de réinstituer le Pacte avec l'autre, présent naguère dans la parole.

\section{INDEX}

Mots-clés : entretien 